Arq. Bras. Med. Vet. Zootec., v.67, n.2, p.405-410, 2015

\title{
Perfil de progesterona em novilhas com diferente atividade luteal e tratadas com implantes vaginais
}

\author{
[Serum progesterone in heifers with different luteal activity treated with vaginal devices] \\ H.L.D. Neri, C.A.C. Fernandes, M.P. Palhão, J.R. Rossi, F.C. Varago \\ Unifenas - Alfenas, MG
}

\begin{abstract}
RESUMO
O dispositivo de liberação de progesterona (DLP) é muito importante em protocolos de Inseminação Artificial em Tempo Fixo (IATF). Representa cerca de $43 \%$ dos custos e é objeto de estudos sobre a eficiência da sua reutilização. No entanto, perfis de liberação de progesterona (P4) em animais com diferentes concentrações endógenas desse esteroide não são claramente descritos. Este estudo teve como objetivo avaliar a concentração sérica de P4 em fêmeas com diferentes situações de atividade luteal, tratadas com DLP novo (1g de P4) por 8 dias. Trinta novilhas mestiças cíclicas foram divididas em três grupos: em G1 e G2, o DLP foi inserido (D0) sete dias após a ovulação induzida. Adicionalmente, 0,15mg de D-cloprostenol foi administrado três dias depois para promover a luteólise em G2. Para G3, o corpo lúteo foi lisado antes da inserção do DLP para que a P4 exógena fosse a única fonte desse hormônio. O sangue foi coletado no D0, D3, D5 e D8, e a P4 avaliada por RIA. Médias de P4 foram comparadas entre os grupos em cada dia e dentro do grupo, entre os dias, utilizando o teste Tukey. Antes da inserção do implante (D0), os níveis de P4 foram, nos grupos, semelhantes em G1 e G2, e superiores a G3 $\left(5,3 \pm 3,1^{\mathrm{a}}\right.$ e $5,3 \pm 1,4^{\mathrm{a}}$ vs $0,6 \pm 0.3^{\mathrm{b}} \mathrm{ng} / \mathrm{mL}$, respectivamente-P $\left.<0,05\right)$. No $\mathrm{D} 3$, ocorreu o mesmo perfil $\left(5,7 \pm 2,6^{\mathrm{a}}\right.$ e $5,4 \pm 2,0^{\mathrm{a}}$ e $3,6 \pm 0.8^{\mathrm{b}} \mathrm{ng} / \mathrm{mL}$, respectivamente para $\mathrm{G} 1$ e $\mathrm{G} 2$ vs G3, $\left.\mathrm{P}<0,05\right)$. Trinta e seis horas (D5) após a PGF, a P4 no G2 caiu para níveis semelhantes aos do grupo G3 e ambos diferiram $(\mathrm{P}<0,05)$ de G1 $\left(3,3 \pm 1,6^{\mathrm{b}}\right.$ vs $2,4 \pm 0,9^{\mathrm{b}}$ e $\left.2,1 \pm 0.7^{\mathrm{b}} \mathrm{ng} / \mathrm{mL}\right)$. Essa diferença se manteve $(\mathrm{P}<0,05)$ em $\mathrm{D} 8\left(3,1 \pm 1,3^{\mathrm{a}}\right.$, $1,8 \pm 0,8^{\mathrm{b}}$ e $\left.1,6 \pm 0.6^{\mathrm{b}} \mathrm{ng} / \mathrm{mL}\right)$. O aumento da $\mathrm{P} 4$ após a inserção (D3 - D0) foi maior $(\mathrm{P}<0,05)$ em G3 que em G1 e G2 $\left(2,8 \pm 0,9^{\mathrm{a}}\right.$ vs $0,4 \pm 1,8^{\mathrm{b}}$ e $\left.0,2 \pm 1.4^{\mathrm{b}} \mathrm{ng} / \mathrm{mL}\right)$. Os animais com maior P4 endógena levam a menor liberação de P4 exógena a partir do DLP. Portanto, os níveis remanescentes de P4 no DLP após o uso dependem da concentração endógena de P4 do animal e possíveis alterações durante a permanência.
\end{abstract}

Palavras-chave: corpo lúteo, dispositivos vaginais, folículos, IATF, reprodução

\begin{abstract}
The progesterone (P4) device is a very important step in the ovulation control in Timed Artificial Insemination (TAI) protocols. It represents about $43 \%$ of the hormone costs, thus it has been the subject of several studies on efficiency of the reused device as an alternative to reduce TAI costs. However, to our knowledge, profiles for P4 release in animals with different endogenous concentrations of P4 are not clearly described. This study aimed to evaluate serum concentration of P4 in females with different ovarian conditions - related to luteal activity - and treated with a new intravaginal device (1g of P4) for 8 days. Thirty normally cyclic crossbred heifers were divided into three groups: for G1 and G2, P4 device was inserted (DO) seven days after ovulation (7 day old $C L)$. Additional PGF ( $0.15 \mathrm{mg}$ of Dcloprostenol) was given three days later to promote luteolysis in the G2 group. For G3, CL was killed before P4 insertion and the exogenous progesterone was the only source of this hormone. Blood samples were collected on D0,D3, D5 and D8 and P4 concentration was measured by radioimmunoassay (RIA). Means for P4 concentration were compared among groups in each day and within the group among days using the Tukey test. Before P4 device insertion (D0), P4 levels were higher $(P<0.05)$ in G1 and G2 when
\end{abstract}

Recebido em 4 de março de 2014

Aceito em 3 de março de 2015

E-mail: humbertoluis.neri@gmail.com 
compared to $G 3$ (5.3 \pm 3.1 and $5.3 \pm 1.4 \mathrm{vs}$. $0.6 \pm 0.3 \mathrm{ng} / \mathrm{mL}$, respectively). Three days later (D3), the same pattern was observed $(5.7 \pm 2.6$ and $5.4 \pm 2.0$ and $3.6 \pm 0.8 n g / m L$, respectively for $G 1$ and G2 vs. G3, $P<0.05)$. Thirty-six hours (D5) after PGF injection (G2), P4 in G2 dropped to levels similar to the G3 group and both differed $(P<0.05)$ from $G 1$ (3.3 \pm 1.6 vs. $2.4 \pm 0.9$ and $2.1 \pm 0.7 \mathrm{ng} / \mathrm{mL}, G 1$ vs. G2 and G3, respectively). There were no differences $(P>0.05)$ among groups on $D 8(3.1 \pm 1.3,1.8 \pm 0.8$ and $1.6 \pm 0.6 \mathrm{ng} / \mathrm{mL}$, respectively, for G1, G2 and G3). Progesterone increase after P4 insertion (D3 - D0) was higher $(P<0.05)$ in $G 3$ compared to $G 1$ and $G 2(2.8 \pm 0.9$ vs. $0.4 \pm 1.8$ and $0.2 \pm 1.4 n g / m L$, respectively). The interpretation was that animals with higher endogenous P4 promote less release of the exogenous P4 from the device. Therefore, the remaining P4 levels from used progesterone devices depend on the physiological condition of the animal at the time of insertion and possible changes during the treatment.

Keywords: corpus luteum, P4 device, follicles, FTAI, reproduction

\section{INTRODUÇÃO}

A crescente necessidade de aprimoramento técnico da atividade pecuária de corte ou de leite para melhorar a eficiência e a rentabilidade das propriedades é motivo de constante estudo e desenvolvimento de tecnologias. Entre as questões de maior relevância, está a eficiência reprodutiva, haja vista que o bom desempenho dessa atividade é determinante para o sucesso financeiro das empresas rurais nesse segmento.

Inserida nesse contexto está a técnica de Inseminação Artificial em Tempo Fixo (IATF), que otimiza a utilização de uma biotécnica de grande importância para melhoramento genético, a inseminação artificial. A IATF permite a indução sincronizada da ovulação das matrizes em momentos pré-determinados e proporciona, entre outras vantagens, a antecipação da concepção com consequente diminuição do intervalo de partos, a concentração dos serviços de monta e dos nascimentos e o ganho genético obtido com o maior número de crias oriundas de touros melhoradores (Bó et al., 2003).

A emergência sincronizada de uma nova onda de desenvolvimento folicular e manutenção de concentrações elevadas de progesterona (P4), simulando as ocorrências fisiológicas do ciclo estral desses animais é importante para o resultado do protocolo (Viana et al., 2008). No Brasil, pela característica de predomínio de matrizes zebuínas ou cruzadas, normalmente sem atividade luteal no momento da IATF, a utilização de dispositivos de liberação de P4 é ainda mais importante. A P4, além de ser essencial para a eficácia do protocolo, é de grande importância por representar em torno de $43 \%$ do custo total do processo. A utilização de dispositivos se deve à necessidade de concentrações elevadas desse esteroide por longos períodos. Os implantes liberam $\mathrm{P} 4$ a favor de gradiente de concentração; nesse caso, a concentração endógena de $\mathrm{P} 4$ pode influenciar na quantidade de progesterona liberada em cada implante e, como consequência, na quantidade remanescente após o uso. Nesse contexto, alguns estudos vêm sendo realizados para viabilizar a reutilização desse produto em virtude da possibilidade de alguma quantidade de progesterona residual após sua reutilização. No entanto, os resultados são controversos e não foi descrito de forma convincente o padrão de liberação da $\mathrm{P} 4$ de implantes utilizados em vacas em diferentes etapas do ciclo estral. O objetivo deste trabalho foi avaliar o perfil de liberação de progesterona de dispositivos vaginais em animais com diferentes situações de P4 endógena.

\section{MATERIAL E MÉTODOS}

Foram utilizadas 30 novilhas com cruzamento racial indefinido, entre 24 e 30 meses de idade, criadas sob o regime extensivo, mantidas em pastagem de brachiaria decumbens, com fornecimento ad libidum de água e sal mineral comercial. Os animais foram previamente avaliados por ultrassonografia transretal (Mindray-2200 - DPS - Equipamentos médicos, com probe linear de $7.5 \mathrm{MHz}$ ) para verificar a atividade luteal. Somente animais contendo corpo lúteo na avaliação, ou seja, cíclicos, foram incluídos nos protocolos de pré-sincronização.

Os animais foram divididos em três tratamentos: em G1 e G2, as fêmeas tiveram a ovulação sincronizada e o dispositivo de $\mathrm{P} 4$ foi inserido (D0) sete dias após a ovulação (CL com 7 dias de idade). Visando à redução da P4 endógena em $\mathrm{G} 2$, uma dose de luteolítico $(0,15 \mathrm{mg}$ de $\mathrm{D}$ cloprostenol) foi administrada três dias depois da 
inserção do implante (D3) para promover a luteólise.

As fêmeas do tratamento G3 iniciaram o tratamento sem $\mathrm{P} 4$ endógena. O CL foi lisado e nova ovulação bloqueada antes da inserção de $\mathrm{P} 4$, garantindo que progesterona exógena fosse a única fonte desse hormônio. Para que as concentrações iniciais endógenas de P4 fossem as descritas acima nos respectivos grupos, tratamentos de pré-sincronização foram utilizados para G1 e G2 (Fig. 1) e um segundo tratamento para G3 (Fig. 2).

\section{G1 e G2}

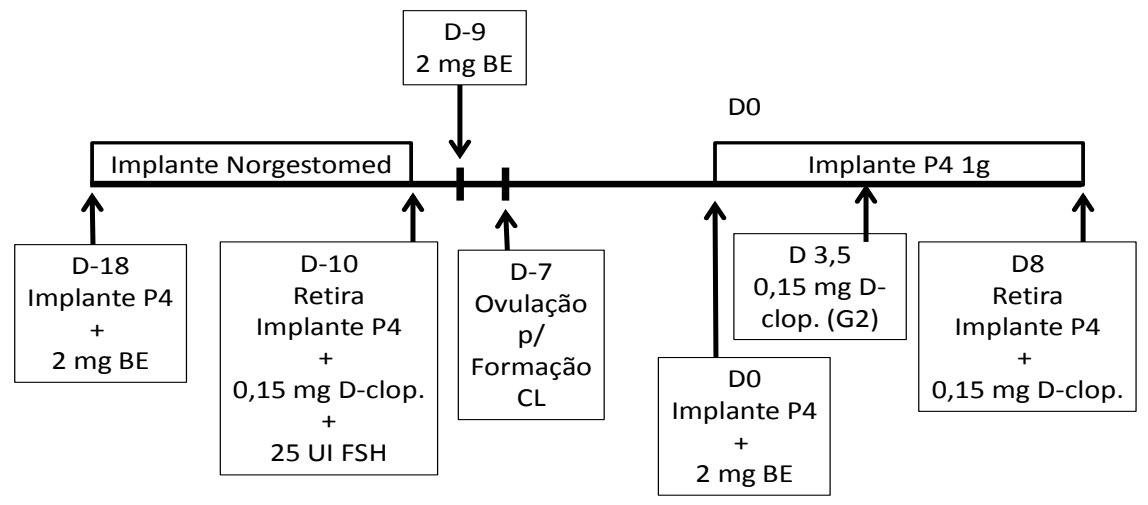

Figura 1. Protocolo de pré-sincronização e de tratamento dos animais do G1 e G2 e momento da aplicação do luteolítico no G2.

G3

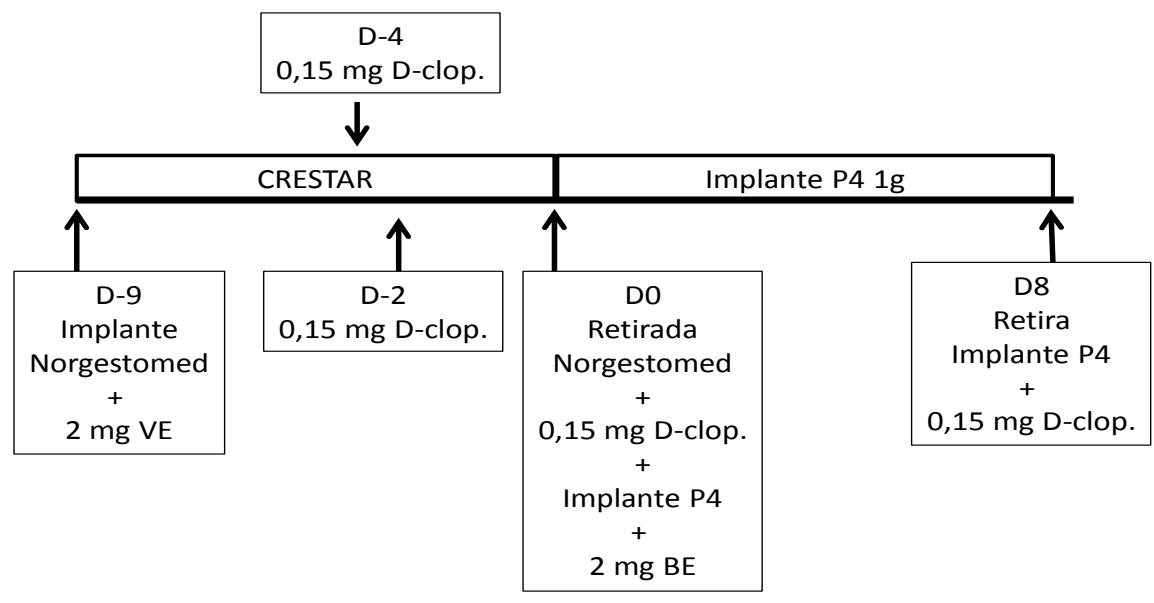

Figura 2. Protocolo de pré-sincronização e de tratamento dos animais do G3. 
Para a obtenção do perfil circulante de P4, foram coletadas amostras de sangue pela punção da veia ou artéria coccígea no D0, imediatamente antes da colocação do implante, no D 3,5 (momento da aplicação do D-cloprostenol nos animais do tratamento G2), no D5 e no D8. As amostras foram mantidas refrigeradas a $4^{\circ} \mathrm{C}$ até o momento da centrifugação. Em um intervalo menor que 6 horas após a coleta, todas foram centrifugadas a $600 \mathrm{G}$ por 10 minutos; em seguida, o soro foi recuperado e estocado a $-20^{\circ} \mathrm{C}$ em frascos eppendorf. Posteriormente foram avaliadas pela técnica de radioimunoensaio, conforme descrito por Fernandes e Figueiredo (2007).

Os dados foram submetidos ao teste de normalidade. Como se apresentaram normalmente distribuídos, foram submetidos à análise de variância (anova). As médias de concentrações de progesterona nos diferentes dias de coleta foram comparadas entre os tratamentos pelo teste de Tukey, utilizando-se o programa SAEG. As diferenças foram consideradas significativas a $5 \%$ de probabilidade.

\section{RESULTADOS E DISCUSSÃO}

Os animais dos grupos G1 e G2, que iniciaram o estudo com CL, apresentaram-se ao início do protocolo com quantidades maiores de $\mathrm{P} 4$ endógena em relação ao grupo G3 $(\mathrm{P}<0,05)$. Adicionalmente, não houve diferença na concentração de P4 entre G1 e G2 (P>0,05), demonstrando a homogeneidade dessa variável entre esses grupos.

Conforme relatado por Inskeep (2004), a concentração de progesterona deve ser maior que $1 \mathrm{ng} / \mathrm{mL}$ durante todo o protocolo, resultado semelhante ao descrito por Almeida et al. (2006), que esclarecem a necessidade de P4 em níveis como os luteais durante o tratamento. Viana et al. (2008) caracterizam a utilização dos implantes como uma alternativa viável para manter elevadas concentrações de $\mathrm{P} 4$ por longos períodos. Lavy et al. (2006) corroboram esse dado ao descreverem os implantes como alternativa quando se desejam níveis elevados de progesterona.

Em todos os grupos durante o período de avaliação, a concentração de $\mathrm{P} 4$ esteve acima do mínimo necessário para os eventos fisiológicos (Fig. 3). Com isso, constata-se que na primeira utilização os implantes mantêm os níveis de P4, independentemente da concentração endógena existente nos animais.

A P4 média apresentou elevação expressiva $(\mathrm{P}>0,05)$ após a colocação dos implantes apenas no G3 (Tab. 1), que pode ser notada pela diferença entre a concentração plasmática de D0 e D3. Considerando a concentração inicial de P4 e o perfil após a colocação dos dispositivos (Fig. 3 ), percebe-se claramente que a liberação de progesterona do implante dependeu da quantidade desse hormônio endógeno e foi diretamente proporcional ao gradiente de concentração, ou seja, diferença entre P4 no implante e no animal.

Esse fenômeno é equivalente ao descrito por Brunton e Parker (2008), denominado difusão passiva, um mecanismo de farmacocinética importante para o tratamento com esteroides, que caracteriza o transporte do ativo de acordo com um gradiente de concentração. Situação semelhante foi observada neste estudo, em que as fêmeas dos grupos G1 e G2 que apresentavam concentrações luteais de progesterona no início do protocolo não demonstraram elevações nas concentrações $(\mathrm{P}>0,05)$ após a colocação do dispositivo. Ao contrário, nos animais do tratamento G3 que iniciaram o estudo sem P4 endógena, tiveram aumento significativo após a inserção do dispositivo, indicando que os implantes utilizados neste último tratamento liberaram mais hormônio que os dos outros grupos. Rothen-Weinhold et al. (2000) relatam que a mucosa vaginal possui elevada permeabilidade aos esteroides, permitindo rápida troca entre o dispositivo e o organismo, reforçando a viabilidade da difusão passiva como mecanismo de liberação de progesterona a partir de implantes vaginais.

Em resumo, em animais sem corpo lúteo, ou seja, com baixos níveis de progesterona circulante, em que há maior gradiente de concentração entre o implante e o animal, o dispositivo libera maior quantidade do hormônio. Com a maior liberação de progesterona, o implante utilizado nesse tipo de animal, sem CL e com P4 endógena baixa, terá menos P4 remanescente após seu uso. Situação diferente dos implantes utilizados em G1 e G2, que 
liberaram para o animal quantidades menores desse esteroide. Com isso, pode-se especular que implantes utilizados previamente em animais com diferentes níveis endógenos de P4 possuem quantidades distintas de $\mathrm{P} 4$ remanescentes.

Tabela 1. Perfil de liberação de progesterona (P4) em novilhas com diferentes condições de progesterona endógena (com ou sem CL), tratadas com implante vaginal de $1 \mathrm{~g}$ novo

\begin{tabular}{l|lllll} 
Grupo & D0 & D3 & $D 5$ & $D 8$ & DIF D3-D0 \\
\hline G1 $(\mathrm{n}=10)$ & $5,3 \pm 3,1 \mathrm{a}$ & $5,8 \pm 2,7 \mathrm{a}$ & $3,4 \pm 1,7 \mathrm{a}$ & $3,2 \pm 1,3 \mathrm{a}$ & $0,5 \pm 1,8 \mathrm{~b}$ \\
$\mathrm{G} 2(\mathrm{n}=10)$ & $5,3 \pm 1,4 \mathrm{a}$ & $5,5 \pm 1,9 \mathrm{a}$ & $2,5 \pm 1,0 \mathrm{~b}$ & $1,8 \pm 0,8 \mathrm{~b}$ & $0,2 \pm 1,5 \mathrm{~b}$ \\
$\mathrm{G} 3(\mathrm{n}=10)$ & $0,6 \pm 0,3 \mathrm{~b}$ & $3,6 \pm 0,8 \mathrm{~b}$ & $2,1 \pm 0,7 \mathrm{~b}$ & $1,6 \pm 0,6 \mathrm{~b}$ & $2,8 \pm 1,0 \mathrm{a}$ \\
\hline
\end{tabular}

Letras diferentes na mesma coluna indicam resultados com significância a 5\% de probabilidade.

Os animais dos tratamentos G1 e G2 mantiveram as concentrações de P4 semelhantes e maiores que o G3 até D3, quando os animais do G2 receberam o agente luteolítico. A partir desse dia, houve a queda da P4 endógena em G2 e o perfil se alterou. (Fig. 3).

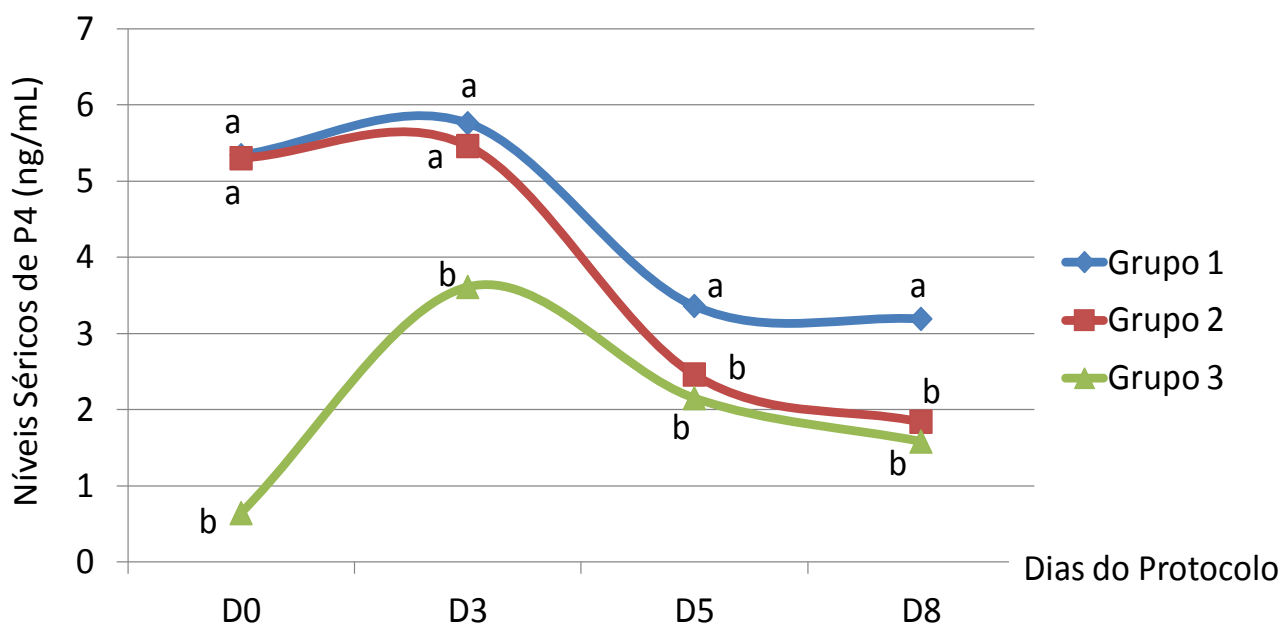

Figura 3. Perfil de liberação de progesterona (P4) em novilhas com diferentes condições de progesterona endógena (com ou sem CL), tratadas com implante vaginal de $1 \mathrm{~g}$ novo (Letras diferentes na mesma avaliação apresentam significância $(\mathrm{P}<0,05))$.

Após a aplicação do luteolítico, os animais com CL do G2 (D 3,5), que apresentavam perfil de progesterona semelhante ao G1, passaram a apresentar perfil de progesterona semelhante ao G3 (Tab. 1). Essa alteração foi percebida em D5, pois a lise do $\mathrm{CL}$ e a queda da P4 endógena necessitam de algumas horas para que ocorram. Estes resultados mostram que a queda da produção de P4 endógena, provocada pelo luteolítico, não é imediatamente compensada pela progesterona do dispositivo, embora Rothen-Weinhold et al. (2000) tenham relatado que as características dos implantes e da mucosa vaginal permitem rápido trânsito, com elevação ou queda das concentrações de P4 em poucas horas após a colocação ou a retirada dos implantes.

De acordo com as variações observadas nas concentrações de P4, nos diferentes animais, nos diferentes dias de avaliação, verifica-se que a quantidade de P4 liberada pelos implantes vaginais depende da condição da concentração endógena dos animais durante o tratamento. 
De acordo com o descrito por Sávio et al. (1993), a P4 não deve atingir concentrações séricas inferiores a $1 \mathrm{ng} / \mathrm{mL}$, a fim de não permitir alterações hormonais que poderiam prejudicar o desenvolvimento folicular e fertilidade. Holness et al. (1981), Fonseca et al. (1983) e Folmam et al. (1990) reforçam tal situação ao relatar que vacas com níveis reduzidos de progesterona durante a fase luteal (abaixo de $1 \mathrm{ng} / \mathrm{mL}$ ), antes da inseminação artificial, apresentam menores índices de fertilidade quando comparadas a vacas com níveis elevados desse hormônio. Assim, com base nos resultados obtidos neste experimento quanto ao perfil de liberação de P4, conclui-se que a utilização de implantes novos é suficiente para suprimir o pico de LH durante o protocolo de IATF, independentemente da condição da P4 endógena dos animais submetidos a sincronização, porém a quantidade de progesterona liberada a partir desses dispositivos é influenciada pela concentração plasmática do hormônio endógeno.

\section{CONCLUSÃO}

Os resultados mostram que a concentração de P4 endógena interfere na liberação de $\mathrm{P} 4$ a partir do dispositivo. Portanto, os níveis remanescentes de P4 no dispositivo de progesterona previamente utilizado dependem da condição fisiológica do animal e possíveis alterações durante $o$ tratamento, quando são utilizados pela primeira vez.

\section{REFERÊNCIAS}

ALMEIDA, A.B.; BERTAN, C.M.; ROSSA, L.A.F. et al. Avaliação da reutilização de implantes auriculares contendo norgestomed associados ao valerato ou benzoato de estradiol em vacas nelore inseminadas em tempo fixo. Braz. J. Vet. Res. Anim. Sci., v.43, p.456-465, 2006.

BÓ, G.A.; BARUSELLI P.S.; MARTÍNEZ M.F. Pattern and manipulation of follicular development in Bos indicus cattle. Anim. Reprod. Sci., v.78, p.307-26, 2003.

BRUNTON, L.L.; PARKER, K.L. Manual of pharmacoloy and therapeutics. 4.ed. Chicago: McGraw-Hill, 2008. p.1254.
FERNANDES, C.A.C.; FIGUEIREDO, A.C.S. Avanços na utilização de prostaglandinas na reprodução de bovinos. Rev. Bras. Reprod. Anim., v.31, p.406-414, 2007.

FOLMAN, Y.; KAIM, M.; HERZ, Z.; ROSENBERG, M. 1. Comparison of methods for synchronization of estrous cycles of dairy cows. 2. Effects of progesterone and parity on conception. J. Dairy Sci., v.73, p.2817-2825, 1990.

FONSECA, F.A.; BRITT, J.H.; MCDANIEL, B.T. et al. Reproductive traits of Holsteins and Jerseys: effects of age, milk yield and clinical abnormalities on involution of cervix and uterus, ovulation, estrous cycles, detection of estrous, conception rate and days open. J. Dairy Sci., v.66, p.1128-1147, 1983.

HOLNESS, D.H.; SPROWSON, G.W.; SHERWARD, C.; GEEL G. Studies on plasma progesterone concentrations and fertility in Friesland dairy cows during the post-partum period. J. Anim. Sci., v.97, p.649-655, 1981.

INSKEEP, E.K. Preovulatory, postovulatory, and postmaternal recognition effects of concentrations of progesterone on embryonic survival in the cow. J. Anim. Sci., v.82, p.E24E39, 2004.

LAVY, E.; STEINMAN, A.; SOBACK, S. Oral controlled-release formulation in veterinary medicine. Crit. Rev. Ther. Drug Carrier Syst., v.23, p.165-204, 2006.

ROTHEN-WEINHOLD, A.; GURNEY, R.; DAHN, M. Formulation and technology aspects of controlled drug delivery in animals. Pharmacol. Sci. Technol. Today., v.3, p.222-231, 2000.

SAVIO, J.D.; THATCHER, W.W.; MORRIS, G.R. et al. Effects of induction of low plasma progesterone concentrations with a progesteronereleasing intravaginal device on follicular turnover and fertility in cattle. J. Reprod. Fertilit. v.98, p.77-84, 1993.

VIANNA, G.N.O; KOZICKI, L.E.; WEISS, R.R. et al. Comparação de diferentes protocolos para a sincronização de estro e inseminação artificial em tempo fixo em vacas da raça nelore em anestro pós-parto. Arch. Vet. Sci., v.13, p.247-254, 2008. 